\title{
UNILATERAL PROPTOSIS OF UNEXPLAINED ORIGIN PART II*
}

\author{
BY \\ ALY MORTADA \\ Faculty of Medicine, Cairo University, Cairo, Egypt.
}

ORBITAL exploration has shown that the two commonest causes of unilateral proptosis of unexplained origin are small cavernous haemangiomata and blood cysts in the muscle cone space not extending to the orbital apex (Mortada, 1962). The rare cases in which orbital exploration reveals no lesions and the proptosis continues to progress should be kept under observation. The four cases to be described in this paper show how repeated skull $x$ rays and ocular and orbital examinations determine the site and nature of the tumour and biopsy determines the pathology of the condition.

\section{Case Reports}

In the four cases to be described the general condition of the patients was good. There was no important family history. The skull shape was normal and the face did not show any haemangioma or other abnormality. There were no signs of endocrine disturbances, no enlargement of lymph glands or of the thyroid gland, liver, or spleen. Chest examination revealed no abnormality. There were no allergic manifestations or signs of vitamin deficiency. The skin did not show pigmentation or nodules. The nervous system, temperature, pulse, blood pressure, urine, and faeces were normal. The blood total and differential counts, erythrocyte sedimentation rate, and bleeding and clotting times were normal. The blood Wassermann reaction and Casoni's test for hydatid were negative and the tuberculin test was usually negative. Basal metabolic rate and 131iodine uptake tests were normal.

At the time of first examination the ears, nose, throat, and nasopharynx were normal. Postero-anterior, oblique, lateral and base skull $x$ rays and those of other parts of the skeleton were normal. There was no history of trauma and no acute inflammatory local signs such as oedema, redness of the lids, or conjunctival chemosis.

The proptosis was painless, not intermittent, not pulsating, slight in degree (about $19 \mathrm{~mm}$.), and of about one year's duration. Apart from the proptosis the eyes were normal. There was no limitation of ocular movements, ptosis, or unilateral myopia. The fundi were normal and there was no visual deterioration. No orbital mass could be palpated through the lids or conjunctival fornix or after general anaesthesia. 
As the cause of the proptosis was unknown, the orbit was explored by lateral orbitotomy (Knapp, 1874; Reese, 1935). Palpation of the posterior surface of the globe, optic nerve, four orbital walls, and intermediate areas revealed no orbital lesion, and the cause of the proptosis was unexplained. About one year later, however, the patients returned for examination and the following discoveries were made.

Case 1, a 35-year-old woman, complained of left proptosis of 2 years' duration. The left eye showed deviation downwards and outwards and proptosis of $30 \mathrm{~mm}$. Hertel (Right side $16 \mathrm{~mm}$.). There was limitation of ocular movements in all directions. The fundus showed optic atrophy. The visual acuity was $1 / 60$. In the upper nasal area of the left orbit there was a palpable soft mass $1 \times 2 \mathrm{~cm}$. extending backwards into the upper part or the orbit.

The right eye was normal, and the visual acuity $6 / 6$.

A postero-anterior skull $x$ ray showed opacity of frontal sinuses, and irregular destruction of the left frontal sinus infero-lateral border, the inner part of the superior orbital margin, and the lesser wing of the sphenoid.

Through an upper nasal fornix conjunctival incision a pink tumour was seen. It was soft, mucoid, and not encapsulated, and emerged in pieces, eroding the bone of the nasal orbital margin, joining the left orbit with the left frontal sinus.

Histological Examination.-There were elongated and stellate cells with round or oval nuclei, scanty cytoplasm, and varying amounts of intercellular mucoid material and collagen tissue. The picture was consistent with the diagnosis of a fibromyxoma of the frontal sinus.

Case 2, a 33-year-old woman, complained of left proptosis of 2 years' duration. The left eye showed ptosis, deviation downwards and outwards, and proptosis of $30 \mathrm{~mm}$. (Right side $17 \mathrm{~mm}$.). Ocular movements inwards and downwards were limited. The fundus showed optic atrophy. There was no palpable orbital mass. The visual acuity was hand movements.

The right eye was normal, with normal fundus and visual acuity $6 / 9$.

A postero-anterior skull $x$ ray showed destruction of both left lesser wings and the adjacent part of the greater wing of the sphenoid. The left superior orbital fissure could not be seen. A lateral skull $x$ ray view showed destruction of the posterior clinoid processes and dorsum sellae with deepening of the floor of the sella turcica. The skull base view showed destruction of the base of the skull in the left middle cranial fossa involving the great wing of the sphenoid, and the apex of the petrous part of the temporal bone, with widening of the oval and spinous foramina.

Orbital exploration disclosed in the apex of the orbit a pink, very soft, friable tumour $2 \times 2 \mathrm{~cm}$. emerging in pieces.

Histological Examination.-There were clusters and acini of epithelial cells in a myxomatoid matrix. The picture was that of a malignant mixed salivary gland tumour of the sphenoid sinus.

Case 3, a 29-year-old man, complained of right proptosis of 2 years' duration. The right eye deviated downwards and outwards, with proptosis $20 \mathrm{~mm}$. (Left side $16 \mathrm{~mm}$.), and limitation of ocular movements upwards and inwards. The fundus was normal and the visual acuity 6/12. Above the inner canthus, pushing the medial part of the upper lid forwards, there was a cystic swelling $1 \times 1 \mathrm{~cm}$. extending backwards into the upper nasal quadrant of the orbit.

The left eye was normal, visual acuity $6 / 9$. 
Postero-anterior and right oblique $x$ rays of the skull showed a bony defect in the medial side of the superior orbital margin. A rounded defined area was seen encroaching upon the right frontal sinus and orbital cavity.

Orbital exploration disclosed a bluish cystic swelling occupying the medial upper side of the orbit continuous with the right frontal sinus. The cyst contained a yellowishgreen thick sterile fluid.

Histological Examination.-The cyst wall showed subepithelial mononuclear infiltration. The picture was that of a frontal mucocele.

Case 4, a 25-year-old man, complained of right proptosis of 2 years' duration. The right eye showed ptosis, deviation downwards, and proptosis of $25 \mathrm{~mm}$. (Left side $17 \mathrm{~mm}$.). There was limitation of ocular movements upwards and downwards. The fundus showed post-papilloedemic optic atrophy. The visual acuity was no perception of light. A palpable hard mass $1 \times 2 \mathrm{~cm}$. was felt in the right orbit between the lower temporal orbital margin and the globe.

The left eye was normal, visual acuity $6 / 9$.

A postero-anterior skull $x$ ray showed a wider right orbital cavity and opacity of the right maxillary and ethmoid sinuses. The lesser and greater wings of the sphenoid on the right side were replaced by an area of irregular bone density with typical sunburst appearance. There was loss of contour of the right superior orbital fissure. A typical sunray appearance was noted also in the lateral and right oblique $x$ rays of the skull, the contour of the right optic canal being lost.

Orbital exploration revealed a hard bony tumour involving the lateral orbital wall.

Histological Examination.-Large spindle-shaped cells with hyperchromatic nuclei with areas of new bone formation were consistent with a diagnosis of osteoblastic osteosarcoma.

For further treatment the four cases were sent to consult the rhinologist and the radiologist.

\section{Discussion}

These four cases show that early tumours of orbital bones or tumours or mucoceles of the accessory nasal sinuses may first present with gradual progressive proptosis without $x$ ray changes or orbital lesions. For about one year the cause of the proptosis remains unexplained, and repeated skull $x$ rays must be undertaken. In a series of 200 cases of exophthalmos reported by Pfeiffer (1943) $x$ rays were diagnostic in 42 per cent. and suggestive in 20 per cent.

Tumours of the frontal, ethmoid, maxillary, or sphenoid accessory nasal sinuses may take the form of osteoma, chondroma, osteoclastoma, fibroma, myxoma, angioma, psammoma, endothelioma, carcinoma, or sarcoma (Duke-Elder, 1952). Mixed lacrimal gland tumours of the nasal sinuses are rare. Tumours developing from the glandular elements of the mucous membrane of the nose and paranasal sinuses constitute only about 10 per cent. of the group of mixed salivary tumours (Reese, 1951a). Mucoceles of the nasal sinuses may affect the frontal, ethmoid, or sphenoid air sinuses.

Reese (1951b) reported an osteogenic sarcoma of orbital bone. After starting at the age of 3 years and causing proptosis of the left eye the tumour 
progressed slowly and proved fatal at age 16 . More cases of orbital osteogenic sarcoma following irradiation for retinoblastoma have been reported than cases arising spontaneously (Cahan, Woodard, Higinbotham, Stewart, and Coley, 1948; Tebbet and Vickery, 1952; Zimmerman and In -1957 ). Some tumours following irradiation have components indist. selable from chondrosarcoma, liposarcoma, or fibrosarcoma. The saliunt feature is the presence of obviously neoplastic cells forming osteoid tissue or bone (Hogan and Zimmerman, 1962).

\section{Summary}

(1) Unilateral proptosis of unexplained origin may be classified after orbital exploration as follows:

(a) Cases due to cavernous haemangiomata or blood cysts in the muscle cone space. Removal of the cause cures the proptosis.

(b) When orbital exploration reveals no abnormality but is followed by a slowly progressive proptosis, repeated skull $x$ rays usually show that the cause is a deep orbital wall bony tumour or a tumour or mucocele of an accessory nasal sinus. The tumour later becomes palpable on orbital exploration and a biopsy reveals its pathological nature.

In both cases the early tumour formation is hidden, in the first in the muscle cone space and in the second in a nasal sinus or a deep orbital bone.

(2) These are the first cases of proptosis due to a frontal sinus fibromyxoma, sphenoid sinus malignant mixed salivary tumour, and sphenoid bone osteoblastic-osteosarcoma to be reported from Egypt.

\section{REFERENCES}

Cahan, W. G., Woodard, H. Q., Higinbotham, N. L., Stewart, F. W., and Coley B. L. (1948). Cancer, 1, 3.

DUKE-ELDER, S. (1952). “Text-book of Ophthalmology", vol. 5, p. 5656. Kimpton, London. Hogan, M. J., and Zimmerman, L. E. (1962). "Ophthalmic Pathology. An Atlas and Textbook", 2nd ed., p. 751 . Saunders, Philadelphia.

KnapP, H. (1874). Klin. Mbi. Augenheilk., $12,439$.

MORTADA, A. (1962). Brit. J. Ophthal., 46, 369.

PFeIFfer, R. L. (1943). Amer. J. Ophthal., 26, 724.

ReESE, A. B. (1935). Arch. Ophthal. (Chicago), 14, 41. (1951a). "Tumors of the Eye", p. 478. Hoeber, New York. (1951b). Ibid., p. 449.

TEBBet, R. D., and VICKERY, R. D. (1952). Amer. J. Ophthal., 35, 811.

Zimmerman, L. E., and INGALLS, R. (1957). Ibid., 32, 417. 